\title{
Large COMPASS polarized solid state target for Drell-Yan physics
}

\author{
J. Koivuniemi*, A. Berlin, F. Gautheron, W. Meyer and G. Reicherz \\ E-mail: jaakko.koivuniemi@cern.ch, alexander.berlin@cern.ch, \\ fabrice.gautherondcern.ch, werner.meyerdcern.ch, \\ reicherzetau.ep1.rub.de \\ Ruhr-University Bochum, Germany
}

\section{N. Doshita, T. Iwata, K. Kondo and G. Nukazuka}

E-mail: norihiro.doshita@cern.ch, tiwata@sci.kj.yamagata-u.ac.jp, kaori.kondo@cern.ch, genki.nukazuka@cern.ch

Yamagata University, Japan

\section{Finger, J. Matoušek and M. Pešek}

E-mail: miroslav.finger@cern.ch, jan.matousek@cern.ch,

michael.pesek@cern.ch

Charles University in Prague, Czech Republic

\section{Y. Kisselev}

E-mail: Yury.Kiselevecern.ch

JINR, Dubna, Russia

In the COMPASS Drell-Yan programme a negative pion beam with momentum of $190 \mathrm{GeV} / \mathrm{c}$ and intensity up to $10^{8}$ particles/s is used. The polarizable solid state $\mathrm{NH}_{3}$ target is placed inside the mixing chamber of a large horizontal helium-3/4 dilution cryostat. A large acceptance superconducting solenoid magnet with a nominal field of $2.5 \mathrm{~T}$ is used to polarize the protons by microwave pumping around electron spin frequency. Due to the space needed by the hadron absorber the target platform had to be moved $2.3 \mathrm{~m}$ upstream from deep inelastic scattering position of run 2011. The upgrade of the target system and the initial commissioning are presented.

XVIth International Workshop in Polarized Sources, Targets, and Polarimetry

14-18 September 2015

Ruhr-University Bochum, Germany

\footnotetext{
* Speaker.
} 


\section{Introduction}

In the SIDIS (Semi-Inclusive-Deep-Inelastic-Scattering) program of COMPASS[1] 160-190 $\mathrm{GeV} / \mathrm{c}$ polarized muon beam is used with longitudinally or transversely polarized ${ }^{6} \mathrm{LiD}$ deuteron or $\mathrm{NH}_{3}$ proton target. For the Drell-Yan experiment the beam intensity of $190 \mathrm{GeV} / \mathrm{c}$ pions goes up to $10^{8} / \mathrm{s}$. The ammonia is radiation hard material[2] and can maintain high nuclear polarization in hadron beam conditions. It has typically $10^{-4}-10^{-3} \mathrm{NH}_{2}$ free radicals/nucleus. High nuclear polarization $0.8-0.9$ can be achieved with dynamic nuclear polarization (DNP) method. Fraction of polarizable nuclei or dilution factor 0.176 is good for a proton target material[3] giving high figure of merit $\sim n_{t} p_{t}^{2} f^{2}$, where $n_{t}$ is the number of nuclei per area seen by the beam, $p_{t}$ is the target polarization and $f$ the dilution factor. The target material inside the mixing chamber of large helium-3/4 dilution cryostat is kept in two consecutive $55 \mathrm{~cm}$ long and $4 \mathrm{~cm}$ diameter cells with separation of $20 \mathrm{~cm}$. The solid ammonia beads were produced at Bochum and irradiated by electron beam in the $20 \mathrm{MeV}$ Bonn linac to create paramagnetic centers. After the irradiation the color of the material is violet and becomes pale with aging. The paramagnetic centers are needed for the DNP on the electron spin frequency shifted by the nuclear spin frequency or about $70 \pm 0.1$ $\mathrm{GHz}$ in $2.5 \mathrm{~T}$ magnetic field. After the polarization build up with $2.5 \mathrm{~T}$ solenoid magnet the field is rotated to $0.63 \mathrm{~T}$ transverse dipole field. In the frozen spin mode during the physics data taking the relaxation of nuclear polarization can be expected to be faster than in the $2.5 \mathrm{~T}$ field.

\section{Operation in hadron beam conditions}

FLUKA $^{1}$ simulations gave an estimated total dose of 100 Gy for 220 days of running for materials far away from beam axis and up to $20000 \mathrm{~Gy}$ for the target material on beam. The radiation damage was tested at the Jülich $45 \mathrm{MeV}$ proton cyclotron with no effect on aramid-epoxy target holder nor on glassfiber-epoxy mixing chamber nor significant change in ruthenium oxide or diode thermometer calibration. In the real 2015 beam conditions small rise in mixing chamber temperature $30-40 \mathrm{mK}$ above the base temperature around $50 \mathrm{mK}$ is seen. Tests done on the NMR Q-meter circuit did not reveal any apparent problem in operation of semiconductors in radiation area. Nevertheless the magnet power supplies, safety system and cryogenic control racks were placed outside of the beam area. The Schneider PLC ${ }^{2}$ for isolation vacuum pump system was installed to the counting room. The National Instruments PXI crate for NMR data acquisition was put to the control room too while the rack with Liverpool Q-meter boxes was moved under the target platform and fixed firmly on the floor due to the presence of stray magnetic field from dipole and solenoid magnets. The top part of the rack was covered with $2 \mathrm{~cm}$ lead plates.

\section{Dilution cryostat}

The helium-3 gas from liquid helium-3/4 mixture is pumped with series of eight Pfeiffer roots blowers with canned motors at typical pumping speed of $0.2 \mathrm{~g} / \mathrm{s}$ at 0.65 mbar inlet pressure. In frozen spin mode the still temperature is about $1.0 \mathrm{~K}$ and pressure 0.42 mbar with flow of 0.07

\footnotetext{
${ }^{1}$ http://www.fluka.org/

${ }^{2}$ CPU TSX P575634M
} 
$\mathrm{g} / \mathrm{s}$ at $0.2 \mathrm{mbar}$ roots inlet pressure. The pumping system has six external and one internal water cooled heat exchanger to remove heat from the helium-3 gas compression. The original damaged copper heat exchangers were replaced by new heat exchangers rebuilt from stainless steel. The pump motor temperatures are monitored with Pt100 sensors during the run to get early warning of a cooling fan failure before the pump system stops automatically. The compressed helium-3 gas goes through four parallel Praxair SG6173 activated charcoal cartridges followed by liquid nitrogen trap with activated charcoal before it returns back to the dilution cryostat. The $\mathrm{LN}_{2}$ trap is filled automatically from 2001 buffer dewar by Siemens S7 300 PLC using three Pt100 temperature sensors inside perforated brass tube to estimate the liquid level. The liquid helium- 4 evaporator at $1.4 \mathrm{~K}$ is pumped with Leybold RUVAC 2001 roots blower and Sogevac SV630 rotary pump with flow of $0.4 \mathrm{~g} / \mathrm{s}$. There is a $3 \mathrm{~m}^{2}$ water cooled heat exchanger between the roots and rotary pumps. In addition the rotary pump has an internal cooling water circuit. About 90001 of helium$3 / 4$ gas mixture is condensed to the dilution cryostat mixing chamber with $10-15 \%$ of helium-3. The compressed gas at roots outlet has typically $20-30 \%$ of helium-4. The horizontal dilution cryostat has $0.1 \mathrm{~m}^{2}$ tubular heat exchanger made from flattened stainless steel pipe on bottom of still followed by series of sintered copper heat exchangers $12 \mathrm{~m}^{2}$ and $0.75 \mathrm{~mm}$ thickness [5, 6]. The cold gas on horizontal and vertical thermal screens is controlled with MKS flow controllers. The isolation vacuum is pumped with Edwards Diffstak Mk2 160/700 diffusion pump and Alcatel 2033 rotary pump.

\section{NMR for polarization measurement}

New PCTFE target cells with perforated $0.75 \mathrm{~mm}$ walls were produced. The perforation holes are $1.2 \mathrm{~mm}$ in diameter with $2 \mathrm{~mm}$ distance in between. On both ends of the cell an internal NMR coil was mounted inside. These coils have $50 \mu \mathrm{m}$ thick PCTFE insulation foil wrapped around them. In between the ends three coils were fixed on the outer surface of the target cells with Teflon tape. Miniature coaxial cable UT-034 (Micro-Coax) was soldered on the NMR coils made of $1 \times 0.25 \mathrm{~mm}$ stainless steel tube bent into rectangular shape with outer dimensions of $50 \mathrm{~mm} \times$ $11 \mathrm{~mm}$. The other end of the coaxial cable was soldered to SMA connector and connected at the vacuum feedthroughs of the target holder.

The frequency of the synthesizer PTS250 (Programmed Test Sources) is swept in $600 \mathrm{kHz}$ window around the proton NMR resonance frequency at $106400 \mathrm{kHz}$ in $2.5 \mathrm{~T}$ field. The Q-meter output signal is followed by an automatic offset voltage subtraction circuit on Yale-card[7]. This signal is connected with $20 \mathrm{~m}$ long cables to 32 channel 16-bit A/D-converter card PXI-6224 and PXI-6254 in a PXI-1042 chassis. A 32 channel digital interface card PXI-6533 is sweeping the synthesizer frequency from a LabVIEW program.

\section{Thermometry}

During the thermal equilibrium calibration of the polarization the superfluid helium- 4 temperature around $1 \mathrm{~K}$ is determined with helium-3 vapor pressure thermometer. The bulb pressure was measured with a MKS Baratron 270B and a Pfeiffer CMR372 capacitive pressure gauges. Two 
Picowatt AVS47 and four AVS46 resistance bridges measure the resistive thermometers in the dilution cryostat. Lakeshore cryogenic temperature monitor LS218 reads diode thermometers above $4 \mathrm{~K}$. Proper cabling and correct positioning of resistance bridge preamplifiers for low temperature measurement is not trivial in the complex COMPASS spectrometer. Nevertheless continuous minimum temperatures around $45 \mathrm{mK}$ in the mixing chamber could be measured when the beam was off. The dilution cryostat instruments are read from Linux computer with Perl script using six USB serial adapters based on PL2303X chip and National Instruments PCI-GPIB card with TNT4882 chip. The data is then published with a DIM-server ${ }^{3}$ written in $\mathrm{C}++[8]$ and recorded at the same time into MySQL and SQLite databases. The electropneumatic valve for dilution cryostat liquid helium transfer is controlled manually with 4-20 mA current loop. There are five motor driven needle valves on the dilution cryostat that are controlled manually from control room. Three from the helium gas/liquid separator for cavity flow and gas/liquid transfer to helium-4 evaporator. The helium-3 flow is controlled with a needle valve with optional bypass on heat exchangers between still and helium-4 evaporator.

\section{Microwave system}

Two $20 \mathrm{~W}$ EIO (extended interaction oscillator) CPI VKE2401 microwave tubes are used to generate the power for pumping the paramagnetic centers during polarization build up. The cavity size of these tubes can be tuned to adjust the frequency while maintaining the necessary conditions for oscillation. Solid state power supplies Varian VPW2838 and CPI VPW2827 produce the -5.6 $--5.8 \mathrm{kV}$ cathode, $+3.1 \mathrm{kV}$ anode and $6.3 \mathrm{~V}$ cathode heater voltages. A $1.51 /$ min closed water cooling circuit removes heat from the tubes. Temperature of the rectangular WR-12 waveguide at outlet of each EIO tube is monitored with a Pt100 sensor for any returning power before the $15 \mathrm{~W}$ directional coupler. The power is split equally into two waveguides with $10 \mathrm{~W}$ magic-T. One of the waveguides has adjustable length while the other is a simple curved waveguide. Power from these two is summed up in $10 \mathrm{~W}$ magic- $\mathrm{T}$ and the output power depends on the phase difference between the two waves. The microwave table has two EIP-548-B Phase Matrix frequency counters, one for the upstream and one for the downstream target cell. A multimeter connected to Millitech DET-12-RPFW0 diode measures the microwave power. After $90^{\circ}$ bend on microwave table the waveguide size is adapted to vertical $1 \mathrm{~m}$ long WR-28 and then to horizontal $10 \mathrm{~m}$ long WR-90. The waveguide loss from the control room to the dilution cryostat is about 6-8 dB. The waveguide size is reduced again at the dilution cryostat flange. A vacuum feedthrough with thin FEP plastic foil is used to pass the microwaves to the two rectangular holes with shallow sloping end angle on the cavity wall. The cylindrical cavity made from $1 \mathrm{~mm}$ copper has internal diameter of 410 $\mathrm{mm}$ and length of $1421 \mathrm{~mm}$ with $280 \mathrm{~mm}$ long conical part at downstream end. It was designed for three target cells and had to be modified ${ }^{4}$ with an internal $363 \mathrm{~mm}$ diameter copper cylinder for the Drell-Yan two target cell experiment. The vertical wall separates the cavity into two halves allowing to use different microwave frequencies for the up- and downstream parts.

\footnotetext{
${ }^{3}$ http://dim.web.cern.ch/

${ }^{4}$ This modification can be reverted.
} 


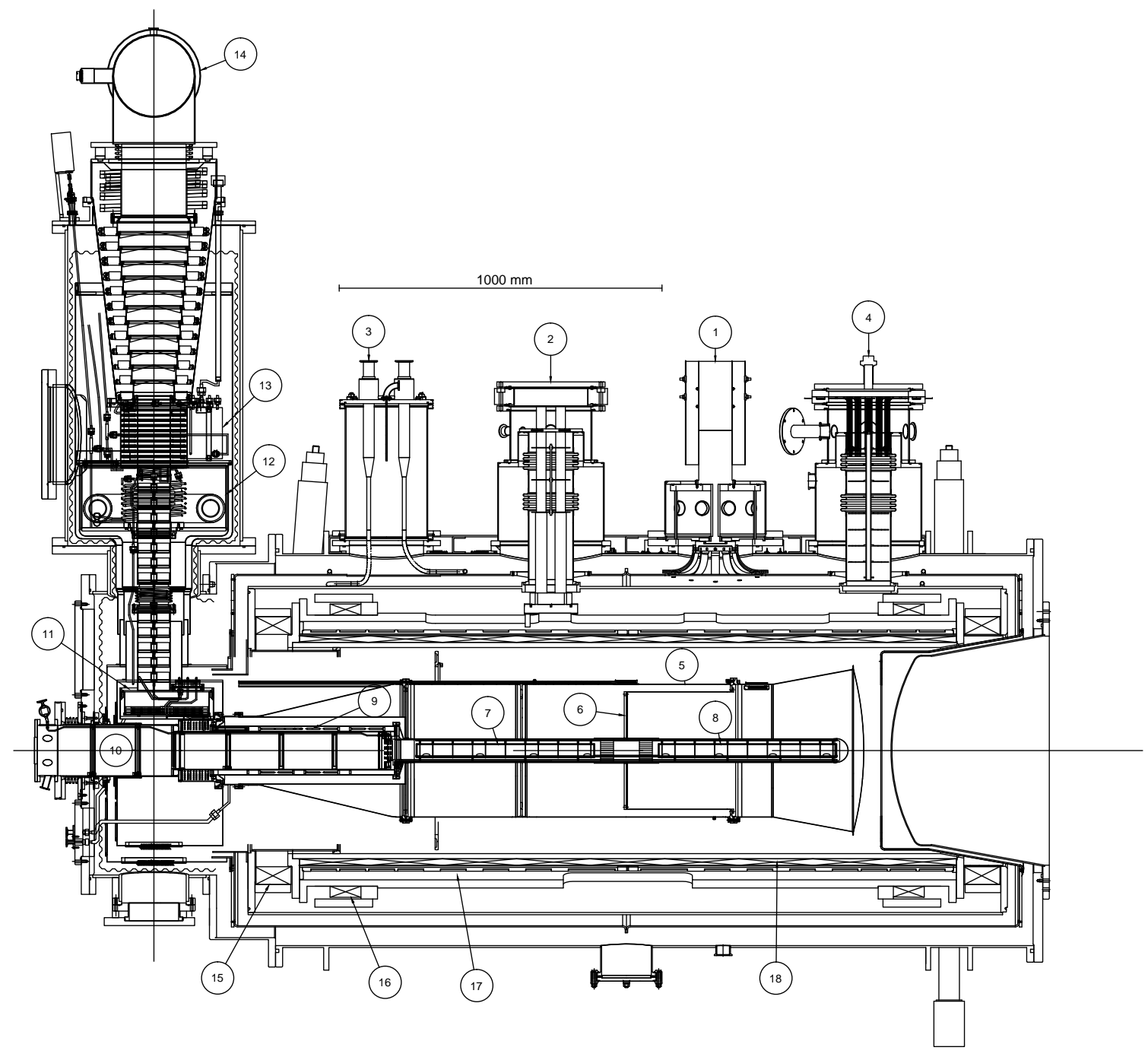

Figure 1: Side view of the COMPASS polarized target in Drell-Yan physics data taking 2015: (1) cryocooler for magnet thermal radiation shield, (2) current lead turret, (3) liquid nitrogen precooling inlet and outlet connections, (4) liquid helium transfer and instrumentation turret, (5) microwave cavity, (6) microwave stopper, (7) upstream and (8) downstream target cell inside aramid-epoxy tube, (9) step heat exchanger, (10) target holder isolation vacuum and radiation shields, (11) still or helium-3 evaporator, (12) helium-4 evaporator, (13) helium-4 gas/liquid separator (14) helium-3 pumping port, (15) upstream end compensation coil, (16) dipole magnet, (17) trim coils and (18) solenoid magnet.

\section{Superconducting magnet}

A superconducting solenoid magnet $(12.4 \mathrm{H})$ with internal bore diameter of $638 \mathrm{~mm}$ and length of $2350 \mathrm{~mm}$ is equipped with 16 shim coils $(0.2-9 \mathrm{H})$ to produce longitudinal magnetic field of 2.5 $\mathrm{T}(647 \mathrm{~A})$ with field homogeneity better than $100 \mathrm{ppm}$ in volume of $40 \mathrm{~mm}$ in diameter and 1300 $\mathrm{mm}$ in length on beam axis. A dipole coil $(2.6 \mathrm{H})$ produces a $0.63 \mathrm{~T}(588 \mathrm{~A})$ transverse field with much worse field uniformity in the target volume. The magnet helium vessel was modified after the longitudinal physics data taking of 2011. Shorts on two trim coils were repaired and several 
design weaknesses addressed by CERN magnet team. New turret was added for liquid nitrogen precooling connections and second turret for CryoMech pulsed tube cryocooler PT60-UL with CP830 air cooled compressor package giving nominal cooling power of $60 \mathrm{~W}$ at $80 \mathrm{~K}$ temperature with $3.3 \mathrm{~kW}$ power consumption and $18 \mathrm{kh}$ service interval. It is used to cool down to $60 \mathrm{~K}$ the thermal screen of magnet cryostat and helps to reduce liquid helium consumption. The thermal shields and current leads are monitored with nine Pt1000 sensors positioned on the cold head and on liquid nitrogen precooling loop. Nine CERN standard temperature bridges are on liquid helium system: one on top and one on bottom of solenoid magnet, three on top, one in middle and one on bottom of the dipole magnet, and two on trim coils.

Due to the large stored energy (3 MJ) in solenoid, propagation of quench is critical for the operation safety. On each coil two Minco heaters $76 \times 76 \mathrm{~mm}^{2}$ were added with a total of 16 new heaters. The solenoid is monitored with four voltage bridges for quench detection in addition to voltage monitoring channels. The dipole magnet energy is smaller $(0.5 \mathrm{MJ})$ and three voltage bridges are used. The magnet safety system opens the circuit breakers from the power converters and fires the heaters in case of sudden voltage rise seen by the quench detection circuit. In addition external diodes, a $1 \Omega$ resistor and cable resistance are used to damp the stored energy in emergency case. The solenoid and dipole power converters are made from two Delta Electronica one quadrant $15 \mathrm{~V} / 400$ A power supplies connected in parallel. These are followed by polarity switch to change the direction of current flow in the magnet. The ramping down of the magnetic field needs to be done by dissipating the stored energy in the external protection diodes.

The magnet monitoring and control is done through CERN UNICOS ${ }^{5}$ system and magnet parameters are published by a DIP ${ }^{6}$ server. Two industrial Windows PCs with ethernet switch are placed in one of the magnet control racks. The longitudinal or transverse field is ramped up by executing one of the preprogrammed scripts in the computer. The dipole and solenoid magnet current leads inside the turret were made of $3 \mathrm{~cm}$ diameter brass tube with 11 smaller diameter tubes tin soldered in. The tube is clamped with bolts at top of the turret and the current is carried through the glassfiber piece by connecting copper braid and brass bolt. The current lead gas flow $0.3 \mathrm{~g} / \mathrm{s}$ going up to $1.3 \mathrm{~g} / \mathrm{s}$ during magnet filling is monitored with a mass flow meter and controlled by an electropneumatic valve. The cold gas is heated up to room temperature with a heater on the return line. The trim coil, voltage tap, thermometer and superconducting level gauge cables are taken into the cold mass with epoxy impregnated feedthroughs from second turret at downstream part of magnet vessel. About 5601 liquid helium volume is re-filled automatically from the same turret. In addition to a superconducting filament level gauge, a differential pressure gauge was used to estimate liquid helium level in dewar. Two +0.5 bar pressure relief valves in parallel with electropneumatic valve controlled by Schneider $\mathrm{PLC}^{7}$ release any excess helium gas pressure with $15 \mathrm{~m}$ long $219.1 \times 3.0 \mathrm{~mm}$ quench vent line going outside of the experimental hall. Additional safety is given by a burst disk specified to higher pressure according to the CERN safety rules.

\footnotetext{
${ }^{5}$ http://unicos.web.cern.ch/

${ }^{6}$ http://cern.ch/dip

${ }^{7}$ TSX P57 5634
} 


\section{Target operation}

The solid target material is filled into the target cells in a liquid nitrogen bath. Then the target holder is lifted up and the mixing chamber volume opened. Small flow of helium shielding gas makes sure that not too much air enters during the loading operation. The target holder is then inserted into the access tube and rapidly pressed with the two clamps from the upstream flange to close in the internal cold indium seal between access tube volume and mixing chamber made of glassfiber epoxy. After this the mixture of air and helium is pumped out by an auxiliary 1000 $\mathrm{m}^{3} / \mathrm{h}$ roots pump system. The thermometer, heater and NMR cables are checked with a multimeter. The target holder isolation vacuum is tested with helium leak detector and at the same time leak tightness of the internal indium joint is tested. The access tube volume is connected to the helium3 pumping line to pump out any possible leaking gas from the mixing chamber. Small helium pressure in the access tube improves the operation of the radiation shields inside the target holder vacuum space.

Liquid helium consumption of the dilution cryostat is about $10 \mathrm{l} / \mathrm{h}$ in frozen spin mode with a helium-3 flow of $24 \mathrm{mmol} / \mathrm{s}$. During the polarization build up this can go up to $16 \mathrm{l} / \mathrm{h}$ due to higher helium-3 flow of $60 \mathrm{mmol} / \mathrm{s}$. The magnet needs about $15 \mathrm{l} / \mathrm{h}$, but this can increase up to 52 $1 / h$ during filling with liquid helium when the transfer valve from the 20001 buffer dewar is open. The liquid nitrogen consumption of the activated charcoal trap in helium-3 circuit is $30 \mathrm{~kg} / \mathrm{d}$.

\section{Acknowledgements}

We wish to thank Angelo Maggiora and Daniele Panzieri for the radiation dose calculations and Vladimir Anosov and Didier Cotte for their support in mechanical installation.

\section{References}

[1] P. Abbon et al., The COMPASS Experiment at CERN, Nucl. Instr. and Meth. A 577 (2007) 455-518 [hep-ex/0703049].

[2] W. Meyer, Ammonia as a polarized solid target material - a review, Nucl. Instr. and Meth. A $\mathbf{5 2 6}$ (2004) 12-21.

[3] St. Goertz, W. Meyer and G. Reicherz, Polarized H, D and ${ }^{3} H e$ targets for particle physics experiments, Prog. Part. Nucl. Phys. 49 (2002) 403-489.

[4] N. Doshita et al., Performance of the COMPASS polarized target dilution refrigerator, Nucl. Instr. and Meth. A 526 (2004) 138-143.

[5] J. Kyynäräinen, The SMC polarized target, Nucl. Instr. and Meth. A 356 (1995) 47-52.

[6] D. Adams et al., The polarized double cell target of the SMC, Nucl. Instr. and Meth. A 437 (1999) 23-67.

[7] K. Kondo et al., Polarization measurement in the COMPASS polarized target, Nucl. Instr. and Meth. A 526 (2004) 70-75.

[8] J. Matoušek and M. Pešek, COMPASS polarized target for Drell-Yan experiment, in proceedings of XVth International Workshop on Polarized Sources, Targets, and Polarimetry, POS (PSTP2013) 009 (2013). 